FIAN/TD-24/09

ITEP/TH-44/09

\title{
Proving AGT relations in the large-c limit
}

\author{
A.Mironov* $^{*}$ and A.Morozov ${ }^{\dagger}$
}

\begin{abstract}
In the limit of large central charge $c$ the 4-point Virasoro conformal block becomes a hypergeometric function. It is represented by a sum of chiral Nekrasov functions, which can also be explicitly evaluated. In this way the known proof of the AGT relation is extended from special to generic set of external states, but in the special limit of $c=\infty$.
\end{abstract}

\section{Introduction}

The AGT relations [1]-15] express conformal blocks [16, 17, 18, of $2 d$ chiral algebras through the Nekrasov functions [19]- 27]. In the case of the Virasoro block with 4 primaries, the both sides of the relation depend on 6 free parameters: five dimensions, four "external" and one "internal" which we parameterize as

$$
\Delta_{i}=\frac{\alpha_{i}\left(\epsilon-\alpha_{i}\right)}{\epsilon_{2} \epsilon_{2}}, \quad i=0, \ldots, 4,
$$

and the central charge, parameterized as $c=1+\frac{6 \epsilon^{2}}{\epsilon_{1} \epsilon_{2}}, \epsilon=\epsilon_{1}+\epsilon_{2}$. The relation states that

$$
\sum_{|Y|=\left|Y^{\prime}\right|} x^{|Y|} \gamma_{\Delta \Delta_{1} \Delta_{2}}(Y) Q_{\Delta}^{-1}\left(Y, Y^{\prime}\right) \gamma_{\Delta \Delta_{3} \Delta_{4}}\left(Y^{\prime}\right)=(1-x)^{-\nu} \sum_{Y, Y^{\prime}} x^{|Y|+\left|Y^{\prime}\right|} Z_{\Delta_{;} \Delta_{2} \Delta_{2} ; \Delta_{3} \Delta_{4}}\left(Y, Y^{\prime}\right)
$$

For notations and other details see [4]. The sum goes over pairs of Young diagrams, but in two different ways: it is diagonal in the number of boxes, $|Y|=\left|Y^{\prime}\right|$ at the l.h.s., while the summation variables are totally free (unconstrained) at the r.h.s. These two expansions are related to boson and fermion representations of more general $\tau$-functions [28, what deserves a more detailed study and discussion. In fact, there are plenty of different questions about the AGT relations, which connect the transcendental and often controversial field of Seiberg-Witten theory [29] and integration over singular instanton moduli spaces with the basic group theory and complex analysis, unified into a difficult but well defined subject of $2 d$ conformal field theory.

In [5, 13, 14 the two limiting cases of (2) were considered: one of large external dimensions, which on the Nekrasov-SW side corresponds to the case of non-conformal (asymptotically free) SYM models, and the other one of large internal dimension $\Delta_{0}$, where the nice Zamolodchikov asymptotic formula [30] allows one to effectively deal with the old controversial case [31] of the instanton calculus in $4 d$ conformal invariant model with $N_{f}=2 N_{c}$ (one can confirm that instanton corrections exist and even odd numbers of instantons contribute, moreover, the end-point of RG flow is described by an elegant modular relation, at least, for $N_{c}=2$ ).

This letter is devoted to one more limit, $c \rightarrow \infty$. In this limit, either $\epsilon_{1} \rightarrow 0$ or $\epsilon_{2} \rightarrow 0$. Then only the chiral Nekrasov functions, i.e.those with $\left(Y, Y^{\prime}\right)=\left(\left[1^{n}\right], \emptyset\right)$ or $\left(\emptyset,\left[1^{n}\right]\right)$ contribute to the r.h.s. of (2), while the l.h.s. becomes a hypergeometric series. In other words, the limit reproduces the situation studied in [7] and [8], where the AGT relations were proved (this is the only case where a complete explicit proof already exists) for the Fateev-Litvinov conformal blocks 32 . The difference is that there restricting the hypergeometricity and chirality came from a special selection of external states, while here it is enough to take, say, $\epsilon_{1} \rightarrow 0$ without constraining external states.

\section{Hypergeometric conformal block}

The fact that

$$
\begin{aligned}
& B_{\Delta ; \Delta_{1} \Delta_{2} \Delta_{3} \Delta_{4}}(x) \stackrel{c \rightarrow \infty}{\longrightarrow}{ }_{2} F_{1}\left(\Delta+\Delta_{1}-\Delta_{2}, \Delta+\Delta_{3}-\Delta_{4} ; 2 \Delta ; x\right)= \\
&=\sum_{n=0}^{\infty} \frac{x^{n}}{n !} \prod_{k=0}^{n-1} \frac{\left(\Delta+\Delta_{1}-\Delta_{2}+k\right)\left(\Delta+\Delta_{3}-\Delta_{4}+k\right)}{2 \Delta+k}
\end{aligned}
$$

*Lebedev Physics Institute and ITEP, Moscow, Russia; mironov@itep.ru; mironov@lpi.ru

†ITEP, Moscow, Russia; morozov@itep.ru 
is known since [33. Still it deserves reminding a simple derivation.

It is instructive to begin with the first terms of the expansion. It can be taken from any standard text-book on $2 d$ conformal field theory [18, 16, in order to have all the notations consistent, we use below [4]. The conformal block is defined in a highly asymmetric way and depends on the order of dimensions. Explicitly,

$$
\begin{gathered}
B(x)=1+x \frac{\left(\Delta+\Delta_{1}-\Delta_{2}\right)\left(\Delta+\Delta_{3}-\Delta_{4}\right)}{2 \Delta}+ \\
+x^{2}\left[\frac{\left(\Delta+\Delta_{1}-\Delta_{2}\right)\left(\Delta+\Delta_{1}-\Delta_{2}+1\right)\left(\Delta+\Delta_{3}-\Delta_{4}\right)\left(\Delta+\Delta_{3}-\Delta_{4}+1\right)}{4 \Delta(2 \Delta+1)}+\right. \\
\left.+\frac{\left[\left(\Delta_{2}+\Delta_{1}\right)(2 \Delta+1)+\Delta(\Delta-1)-3\left(\Delta_{2}-\Delta_{1}\right)^{2}\right]\left[\left(\Delta_{3}+\Delta_{4}\right)(2 \Delta+1)+\Delta(\Delta-1)-3\left(\Delta_{3}-\Delta_{4}\right)^{2}\right]}{2(2 \Delta+1)(2 \Delta(8 \Delta-5)+(2 \Delta+1) c)}\right]+ \\
+\ldots
\end{gathered}
$$

The linear in $x$ term does not depend on $c$ at all, and the $x^{2}$-term depends only through a single entry, $c / 2$ in the Shapovalov matrix. For large $c$, inverse of this matrix has a single non-vanishing element,

$$
Q^{-1} \stackrel{c \rightarrow \infty}{\longrightarrow}\left(\begin{array}{cc}
0 & 0 \\
0 & \frac{1}{4 \Delta(2 \Delta+1)}
\end{array}\right)
$$

Thus, in this limit (4) becomes

$$
\begin{array}{r}
B(x) \stackrel{c \rightarrow \infty}{\longrightarrow} 1+x \frac{\left(\Delta+\Delta_{1}-\Delta_{2}\right)\left(\Delta+\Delta_{3}-\Delta_{4}\right)}{2 \Delta}+ \\
+\frac{x^{2}}{2} \frac{\left(\Delta+\Delta_{1}-\Delta_{2}\right)\left(\Delta+\Delta_{1}-\Delta_{2}+1\right)\left(\Delta+\Delta_{3}-\Delta_{4}\right)\left(\Delta+\Delta_{3}-\Delta_{4}+1\right)}{2 \Delta(2 \Delta+1)}+\ldots
\end{array}
$$

where one can easily recognize the first terms of the hypergeometric series (3).

In the generic $x^{n}$-term the same thing happens: the only elements of the Shapovalov matrix that do not involve $c$-dependent Virasoro commutators, thus being independent of $c$ and, hence, not growing with $c$, are $\left\langle L_{-1}^{n} \Delta \mid L_{-1}^{n}\right\rangle=\left\langle\Delta\left|L_{1}^{n} L_{-1}^{n}\right| \Delta\right\rangle$. Therefore, in general as $c \rightarrow \infty$,

$$
B(x)=\sum_{n=0}^{\infty} x^{n} \frac{\left\langle L_{-1}^{n} V_{\Delta} \mid V_{\Delta_{1}} V_{\Delta_{2}}\right\rangle\left\langle L_{-1}^{n} V_{\Delta} \mid V_{\Delta_{3}} V_{\Delta_{4}}\right\rangle}{\left\langle L_{-1}^{n} \Delta \mid L_{-1}^{n} \Delta\right\rangle}
$$

Note that the limit to pure gauge theories [5, 13] is similarly controlled by the $L_{-1}^{n}$-contributions only. However, particular contributions and the resulting formulas are essentially different from those in the large- $c$ limit.

The triple vertices in the numerator are known from [16, 17, 34, 4, 6]:

$$
\left\langle L_{-1}^{n} V_{\Delta} \mid V_{\Delta_{1}} V_{\Delta_{2}}\right\rangle=\prod_{k=0}^{n-1}\left(\Delta+\Delta_{2}-\Delta_{2}+k\right)
$$

and the second vertex is just the same, with $(1,2) \rightarrow(3,4)$ (the last property is special for the Virasoro symmetry, it is not true in general, see [6, 8] ). The matrix element in the denominator is found by the recursive procedure:

$L_{1} L_{-1}^{n}|\Delta\rangle=L_{-1} L_{1} L_{-1}^{n-1}|\Delta\rangle+2(\Delta+n-1) L_{-1}^{n-1}|\Delta\rangle=\sum_{j=1}^{n} 2(\Delta+n-j) L_{-1}^{n-1}|\Delta\rangle=n(2 \Delta+n-1) L_{-1}^{n-1}|\Delta\rangle$

and

$$
L_{1}^{n} L_{-1}^{n}|\Delta\rangle=n(2 \Delta+n-1) L_{1}^{n-1} L_{-1}^{n-1}|\Delta\rangle=n ! \prod_{k=0}^{n-1}(2 \Delta+k)|\Delta\rangle
$$

This completes the proof of (3). 


\section{Chiral Nekrasov functions}

In the limit $\epsilon_{1} \rightarrow 0$ not only $c \rightarrow \infty$, but also the dimensions (11) grow infinitely: $\Delta_{i} \rightarrow \infty$. In order to avoid this one needs to especially adjust $\alpha$-parameters: choose them so that $\alpha_{i} \sim \epsilon_{1}$. Then, (1) implies that

$$
\Delta_{i}=\frac{\alpha_{i}\left(1+O\left(\epsilon_{1}\right)\right)}{\epsilon_{1}}, \quad \text { i.e. } \quad \alpha_{i}=\epsilon_{1} \Delta_{i}+O\left(\epsilon_{1}^{2}\right)
$$

This behavior of $\alpha_{i}$, in particular, means that one can neglect the $U(1)$-factor $(1-x)^{-\nu}$ in (2), because typically $\nu=\frac{2 \alpha_{1} \alpha_{3}}{\epsilon_{1} \epsilon_{2}}=O\left(\epsilon_{1}\right)$.

\subsection{The $x$-linear terms}

We begin with the illustration: the $x$-linear term at the r.h.s. of (2):

$$
x\left(Z_{[1][0]}+Z_{[0][1]}\right)=-x\left(\frac{\prod_{f=1}^{4}\left(a+\mu_{i}\right)}{\epsilon_{1} \epsilon_{2} 2 a(2 a+\epsilon)}+\frac{\prod_{f=1}^{4}\left(-a+\mu_{i}\right)}{\epsilon_{1} \epsilon_{2} 2 a(2 a-\epsilon)}\right)
$$

where parameters $a$ and $\mu_{i}$ are related to $\alpha_{i}$ linearly [1, 4]:

$$
a=\alpha_{0}-\frac{\epsilon}{2}, \quad \mu_{1}=-\frac{\epsilon}{2}+\alpha_{1}+\alpha_{2}, \quad \mu_{2}=\frac{\epsilon}{2}+\alpha_{1}-\alpha_{2}, \quad \mu_{3}=-\frac{\epsilon}{2}+\alpha_{3}+\alpha_{4}, \quad \mu_{4}=\frac{\epsilon}{2}+\alpha_{3}-\alpha_{4},
$$

\subsubsection{The simplest case: $\alpha_{1,2,3,4}=0$}

If we now put $\alpha_{1,2,3,4}=0$, then (12) becomes

$$
-x \frac{a^{2}-\epsilon^{2} / 4}{2 \epsilon_{1} \epsilon_{2}}=x \frac{\alpha_{0}\left(\epsilon-\alpha_{0}\right)}{2 \epsilon_{1} \epsilon_{2}}=\frac{\Delta_{0}}{2}
$$

This is in excellent match with the conformal block side of (2).

We even did not use (11). What is important for us here, however: only one of the two $Z$-functions actually contributes to (12) in this limit. Indeed,

$$
Z_{[1][0]}=-\frac{\left(a^{2}-\epsilon^{2} / 4\right)^{2}}{\epsilon_{1} \epsilon_{2} 2 a(2 a+\epsilon)}=-\frac{\alpha_{0}^{2}\left(\alpha_{0}+\epsilon\right)^{2}}{2 \epsilon_{1} \epsilon_{2} 2 \alpha_{0}\left(2 \alpha_{0}-\epsilon\right)}=\frac{\Delta_{0}}{2}+O\left(\epsilon_{1}\right)
$$

if $\alpha_{0}$ satisfies (11), while

$$
Z_{[0][1]}=-\frac{\left(a^{2}-\epsilon^{2} / 4\right)^{2}}{\epsilon_{1} \epsilon_{2} 2 a(2 a-\epsilon)}=-\frac{\alpha_{0}^{2}\left(\alpha_{0}+\epsilon\right)^{2}}{\epsilon_{1} \epsilon_{2}\left(2 \alpha_{0}-\epsilon\right)\left(2 \alpha_{0}-2 \epsilon\right)}=O\left(\epsilon_{1}\right)
$$

\subsection{2 $\alpha_{1,2,3,4} \neq 0$}

This remains true when $\alpha_{1,2,3,4}$ are switched on. Then, from AGT relations (13) it follows that

$$
\begin{array}{r}
a+\mu_{1}=\alpha_{0}+\alpha_{1}-\alpha_{2}, \\
a+\mu_{2}=\alpha_{0}+\alpha_{1}+\alpha_{2}-\epsilon, \\
\frac{a+\mu_{3}=\alpha_{0}+\alpha_{3}-\alpha_{4}}{\mu_{2}=\alpha_{0}+\alpha_{3}+\alpha_{4}-\epsilon,}, \\
-a+\mu_{1}=-\alpha_{0}+\alpha_{1}-\alpha_{2}+\epsilon, \\
-a+\mu_{2}=-\alpha_{0}+\alpha_{1}+\alpha_{2}, \\
-a+\mu_{3}=-\alpha_{0}+\alpha_{3}-\alpha_{4}+\epsilon, \\
-a+\mu_{2}=-\alpha_{0}+\alpha_{3}+\alpha_{4},
\end{array}
$$

Underlined are the quantities that vanish in the limit of $\epsilon_{1} \rightarrow 0$, combined with the prescription (11). Now one immediately obtains that

$$
Z_{[0][1]}=-\frac{\epsilon^{2} \epsilon_{1}^{2}\left(\Delta_{0}-\Delta_{1}-\Delta_{2}\right)\left(\Delta_{0}-\Delta_{3}-\Delta_{4}\right)+O\left(\epsilon_{1}\right)}{\epsilon_{1} \epsilon_{2}\left(2 \alpha_{0}-\epsilon\right)\left(2 \alpha_{0}-2 \epsilon\right)}=O\left(\epsilon_{1}\right)
$$


and

$$
Z_{[1][0]}=-\frac{\epsilon^{2} \epsilon_{1}^{2}\left(\Delta_{0}+\Delta_{1}-\Delta_{2}\right)\left(\Delta_{0}+\Delta_{3}-\Delta_{4}\right)+O\left(\epsilon_{1}\right)}{\epsilon_{1} \epsilon_{2} 2 \alpha_{0}\left(2 \alpha_{0}-\epsilon\right)}=\frac{\left(\Delta_{0}+\Delta_{1}-\Delta_{2}\right)\left(\Delta_{0}+\Delta_{3}-\Delta_{4}\right)}{2 \Delta}+O\left(\epsilon_{1}\right)
$$

which coincides with (4). Note that of eight factors (17) only the first and third ones provide $\Delta$-dependent contributions to the conformal block in the limit of interest.

\subsection{The $x^{2}$ terms}

In this case, there are five Nekrasov functions:

$$
\begin{array}{r}
\mathcal{Z}_{[2][0]}=\frac{1}{2 ! \epsilon_{1} \epsilon_{2}^{2}\left(\epsilon_{1}-\epsilon_{2}\right)} \cdot \frac{\prod_{r=1}^{4}\left(a+\mu_{r}\right)\left(a+\mu_{r}+\epsilon_{2}\right)}{2 a\left(2 a+\epsilon_{2}\right)(2 a+\epsilon)\left(2 a+\epsilon+\epsilon_{2}\right)}, \\
\mathcal{Z}_{[0][2]}=\frac{1}{2 ! \epsilon_{1} \epsilon_{2}^{2}\left(\epsilon_{1}-\epsilon_{2}\right)} \cdot \frac{\prod_{r=1}^{4}\left(a-\mu_{r}\right)\left(a-\mu_{r}-\epsilon_{2}\right)}{2 a\left(2 a-\epsilon_{2}\right)(2 a-\epsilon)\left(2 a-\epsilon-\epsilon_{2}\right)}, \\
\mathcal{Z}_{[11][0]}=-\frac{1}{2 ! \epsilon_{1}^{2} \epsilon_{2}\left(\epsilon_{1}-\epsilon_{2}\right)} \cdot \frac{\prod_{r=1}^{4}\left(a+\mu_{r}\right)\left(a+\mu_{r}+\epsilon_{1}\right)}{2 a\left(2 a+\epsilon_{1}\right)(2 a+\epsilon)\left(2 a+\epsilon+\epsilon_{1}\right)}, \\
\mathcal{Z}_{[0][11]}=-\frac{1}{2 ! \epsilon_{1}^{2} \epsilon_{2}\left(\epsilon_{1}-\epsilon_{2}\right)} \cdot \frac{\prod_{r=1}^{4}\left(a-\mu_{r}\right)\left(a-\mu_{r}-\epsilon_{1}\right)}{2 a\left(2 a-\epsilon_{1}\right)(2 a-\epsilon)\left(2 a-\epsilon-\epsilon_{1}\right)}, \\
\mathcal{Z}_{[1][1]}=\frac{1}{\epsilon_{1}^{2} \epsilon_{2}^{2}} \cdot \frac{\prod_{r=1}^{4}\left(a+\mu_{r}\right)\left(a-\mu_{r}\right)}{\left(4 a^{2}-\epsilon_{1}^{2}\right)\left(4 a^{2}-\epsilon_{2}^{2}\right)}
\end{array}
$$

In order to estimate them, one also needs to complement (17) by

$$
\begin{aligned}
& a+\mu_{1}+\epsilon_{2}=\alpha_{0}+\alpha_{1}-\alpha_{2}+\epsilon_{2}, \\
& \frac{a+\mu_{2}+\epsilon_{2}=\alpha_{0}+\alpha_{1}+\alpha_{2}-\epsilon_{1}}{a+\mu_{3}+\epsilon_{2}=\alpha_{0}+\alpha_{3}-\alpha_{4}+\epsilon_{2},} \\
& \underline{a+\mu_{2}+\epsilon_{2}=\alpha_{0}+\alpha_{3}+\alpha_{4}-\epsilon_{1}},
\end{aligned}
$$

where again underlined are the quantities that vanish in the limit of $\epsilon_{1} \rightarrow 0$, combined with prescription (11).

Combining these formulas with prescription (11) and the AGT relations (13), one obtains

$$
\begin{gathered}
\mathcal{Z}_{[2][0]}=O\left(\epsilon_{1}\right) \\
\mathcal{Z}_{[0][2]}=O\left(\epsilon_{1}\right) \\
\mathcal{Z}_{[11][0]}=-\frac{1}{2 ! \epsilon_{1}^{2} \epsilon_{2}\left(\epsilon_{1}-\epsilon_{2}\right)} \cdot \\
\frac{\epsilon^{4} \epsilon_{1}^{4}\left(\Delta_{0}+\Delta_{1}-\Delta_{2}\right)\left(\Delta_{0}+\Delta_{3}-\Delta_{4}\right)\left(\Delta_{0}+\Delta_{1}-\Delta_{2}+1\right)\left(\Delta_{0}+\Delta_{3}-\Delta_{4}+1\right)+O\left(\epsilon_{1}\right)}{\left(2 \alpha_{0}-\epsilon\right)\left(2 \alpha_{0}-\epsilon_{2}\right) 2 \alpha_{0}\left(2 \alpha_{0}+\epsilon_{1}\right)}= \\
=\frac{1}{2 !} \cdot \frac{\left(\Delta_{0}+\Delta_{1}-\Delta_{2}\right)\left(\Delta_{0}+\Delta_{3}-\Delta_{4}\right)\left(\Delta_{0}+\Delta_{1}-\Delta_{2}+1\right)\left(\Delta_{0}+\Delta_{3}-\Delta_{4}+1\right)}{2 \Delta_{0}\left(2 \Delta_{0}+1\right)}+O\left(\epsilon_{1}\right), \\
\mathcal{Z}_{[0][11]}=O\left(\epsilon_{1}^{2}\right) \\
\mathcal{Z}_{[1][1]}=O\left(\epsilon_{1}\right)
\end{gathered}
$$

which reproduces with (4).

\subsection{Selection rule in the general case}

In the generic case, factors in the numerator that are small in the limit under consideration are underlined in the lists (17) and (25). To each of them one can also add any number of $\epsilon_{1}$, but not $\epsilon_{2}$. Similarly, in the denominator the factors

$$
2 a+\epsilon_{2}+k \epsilon_{1}=2 \alpha_{0}+k \epsilon_{1}
$$

are the only vanishing in this limit among all $2 a+k \epsilon_{1}+l \epsilon_{2}$. 
Nekrasov functions. At the next step we need an explicit expression for generic $Z_{Y, Y^{\prime}}$ :

$$
\mathcal{Z}_{Y, Y^{\prime}}=\frac{\eta\left(Y, Y^{\prime}\right)}{\xi\left(Y, Y^{\prime}\right)}
$$

where for any ordered pair of Young diagrams $Y$ and $Y^{\prime}$

$$
\begin{aligned}
\eta\left(Y, Y^{\prime}\right) & =\prod_{(i, j) \in Y} \prod_{\alpha=1}^{4}\left(\phi\left(a_{1}, i, j\right)+\mu_{\alpha}\right) \prod_{\left(i^{\prime}, j^{\prime}\right) \in Y^{\prime}} \prod_{\alpha=1}^{4}\left(\phi\left(a_{2}, i^{\prime}, j^{\prime}\right)+\mu_{\alpha}\right) \\
\xi\left(Y, Y^{\prime}\right) & =\prod_{(i, j) \in Y} E\left(a_{1}-a_{1}, Y, Y^{\prime}, i, j\right)\left(\epsilon-E\left(a_{1}-a_{1}, Y, Y, i, j\right)\right) \times \\
& \times \prod_{(i, j) \in Y} E\left(a_{1}-a_{2}, Y, Y^{\prime}, i, j\right)\left(\epsilon-E\left(a_{1}-a_{2}, Y, Y^{\prime}, i, j\right)\right) \times \\
& \times \prod_{\left(i^{\prime}, j^{\prime}\right) \in Y^{\prime}} E\left(a_{2}-a_{1}, Y^{\prime}, Y, i^{\prime}, j^{\prime}\right)\left(\epsilon-E\left(a_{2}-a_{1}, Y^{\prime}, Y, i^{\prime}, j^{\prime}\right)\right) \times \\
& \times \prod_{\left(i^{\prime}, j^{\prime}\right) \in Y^{\prime}} E\left(a_{2}-a_{2}, Y^{\prime}, Y^{\prime}, i^{\prime}, j^{\prime}\right)\left(\epsilon-E\left(a_{2}-a_{2}, Y^{\prime}, Y^{\prime}, i^{\prime}, j^{\prime}\right)\right),
\end{aligned}
$$

$a_{2}=-a_{1}$ and

$$
\begin{array}{r}
\phi(a, i, j)=a+\epsilon_{1}(i-1)+\epsilon_{2}(j-1) \\
E\left(a, Y, Y^{\prime}, i, j\right)=a+\epsilon_{1}\left(k_{j}^{T}(Y)-i+1\right)-\epsilon_{2}\left(k_{i}\left(Y^{\prime}\right)-j\right)
\end{array}
$$

We now need to find all the elementary factors in this product, which vanish as $\epsilon_{1}, \alpha_{i} \rightarrow 0$.

Numerator. With the numerator it is simple: we should find all the terms of the form $\prod\left(a+\mu_{f}+k \epsilon_{1}\right)$, $\prod\left(a+\mu_{f}+\epsilon_{2}+k \epsilon_{1}\right)$ and $\prod\left(-a+\mu_{f}+k \epsilon_{1}\right)$, i.e. those which have either $i=1$ or $i=2$ or $i^{\prime}=1$ in (32). Each such product contributes $\epsilon_{1}^{2}$, thus the numerator goes to zero as

$$
\eta\left(Y, Y^{\prime}\right) \sim \epsilon_{1}^{2\left[\#(i=1)+\#(i=2)+\#\left(i^{\prime}=0\right)\right]}
$$

$a$-independent contribution to the denominator. The denominator contains factors of two different types: independent of $a$ and dependent on $a$. Contributions of the first type come from the first and the last line in (31). The factor $E(0, Y, Y, i, j)=\epsilon_{1}\left(k_{j}^{T}-i+1\right)-\epsilon_{2}\left(k_{i}(Y)-j\right)$ does not contain $\epsilon_{2}$ and thus vanishes when $\epsilon_{1} \rightarrow 0$ : if $j=k_{i}(Y)$, i.e. when the box $(i, j) \in Y$ is at the left edge of a row. The number of such elements is exactly the same as the number of rows, i.e. is equal to $k_{1}^{T}(Y)=\#(l=0)$. Another factor in the first line of (31) is $\epsilon-E(0, Y, Y, i, j)=-\epsilon_{1}\left(k_{j}^{T}-i\right)+\epsilon_{2}\left(k_{i}(Y)-j+1\right)$, it would vanish as $\epsilon_{1} \rightarrow 0$ if $j=k_{i}(Y)+1$, i.e. if the box is beyond the diagram. Thus, this factor never vanishes in the limit of interest. Taking into account both diagrams $Y$ and $Y^{\prime}$, the contribution of the $a$-independent terms to denominator is $\sim \epsilon_{1}^{\#(i=1)+\#\left(i^{\prime}=1\right)}$.

$a$-dependent contribution to the denominator. Contributions which depend on $a$ come from the second and third lines in (31). The factor $E\left(2 a, Y, Y^{\prime}, i, j\right)=2 a+\epsilon_{1}\left(k_{j}^{T}(Y)-i+1\right)-\epsilon_{2}\left(k_{i}\left(Y^{\prime}\right)-j\right)$ vanishes in the limit iff the coefficient of $\epsilon_{2}$ is unity, i.e. iff $j=k_{i}\left(Y^{\prime}\right)+1$. This can happen whenever the heights of rows of the two diagrams are such that $k_{i}(Y) \geq k_{i}\left(Y^{\prime}\right)+1$. Similarly, $\epsilon-E\left(2 a, Y, Y^{\prime}, i, j\right)=-2 a-$ $\epsilon_{1}\left(k_{j}^{T}(Y)-i\right)+\epsilon_{2}\left(k_{i}\left(Y^{\prime}\right)-j+1\right)$ vanishes in the limit when the coefficient in front of $\epsilon_{2}$ is -1 , i.e. if $j=k_{i}\left(Y^{\prime}\right)+2$, what can happen whenever $k_{i}(Y) \geq k_{i}\left(Y^{\prime}\right)+2$. The two other factors from the third line can be analyzed in the same way: $E\left(-2 a, Y^{\prime}, Y, i^{\prime}, j^{\prime}\right)=-2 a+\epsilon_{1}\left(k_{j}^{T}\left(Y^{\prime}\right)-i^{\prime}+1\right)-\epsilon_{2}\left(k_{i}(Y)-j^{\prime}\right)$ vanishes in the limit iff $k_{i}(Y)-j^{\prime}=1$, i.e. $j=k_{i}(Y)-1$, what is possible whenever $k_{i}\left(Y^{\prime}\right) \geq k_{i}(Y)-1 \geq 1$. Finally, $\epsilon-E\left(-2 a, Y^{\prime}, Y, i^{\prime}, j^{\prime}\right)=2 a-\epsilon_{1}\left(k_{j}^{T}\left(Y^{\prime}\right)-i^{\prime}\right)+\epsilon_{2}\left(k_{i}(Y)-j^{\prime}+1\right)$ vanishes in the limit iff $k_{i}(Y)-j^{\prime}+1=1$, i.e. $j^{\prime}=k_{i}(Y)$, what is possible whenever $k_{i}\left(Y^{\prime}\right) \geq k_{i}(Y) \geq 1$. Clearly, one gets complementary sets of 
constraints: $k_{i}(Y) \geq k_{i}\left(Y^{\prime}\right)+1$ and $1 \leq k_{i}(Y) \leq k_{i}\left(Y^{\prime}\right)$, either one or the other is true, provided $k_{i}(Y) \geq 1$; $k_{i}(Y) \geq k_{i}\left(Y^{\prime}\right)+2$ and $2 \leq k_{i}(Y) \leq k_{i}\left(Y^{\prime}\right)+1$, again either one or the other is true, this time provided $k_{i}(Y) \geq 2$. This means that in the limit of interest the $a$-dependent part of denominator vanishes as $\epsilon_{1}$ in the power, equal to twice the number of rows in $Y$ of the height two or more plus the number of rows in $Y$ of the height exactly one, i.e. as $\epsilon_{1}^{\#(i=1)+\#(i=2)}$.

$\epsilon_{1}$-dependence of the Nekrasov function: the result. Putting all contributions together, one obtains

$$
Z_{Y, Y^{\prime}} \sim \frac{\epsilon_{1}^{2\left(\#(i=1)+\#(i=2)+\#\left(i^{\prime}=1\right)\right)}}{\epsilon_{1}^{\#(i=1)+\#\left(i^{\prime}=1\right)} \epsilon_{1}^{\#(i=1)+\#(i=2)}}=\epsilon_{1}^{\#(i=2)+\#\left(i^{\prime}=1\right)}
$$

Thus the pair $\left(Y, Y^{\prime}\right)$ contributes at $\epsilon_{1} \rightarrow 0$ iff $\#(i=2)=1$ and $\#\left(i^{\prime}=1\right)=0$. This means that $Y^{\prime}$ should have no rows at all, i.e. be an empty diagram, while $Y$ should have only height-one rows,i.e. $Y=\left[1^{|Y|}\right]$. In other words, we proved that the only Nekrasov functions which do not vanish in the limit are the chiral ones with $\left(Y, Y^{\prime}\right)=\left(\left[1^{|Y|}\right], 0\right)$.

\subsection{Sum of the chiral functions}

It now remains to evaluate these chiral diagrams. This is simple:

$$
\begin{array}{r}
Z_{\left[1^{n}\right][0]}=\frac{(-)^{n}}{n !\left(\epsilon_{1} \epsilon_{2}\right)^{n}} \prod_{i=1}^{n} \frac{\prod_{f=1}^{4}\left(a+\mu_{f}+(i-1) \epsilon_{1}\right)}{\left(2 a+\epsilon+(n-i) \epsilon_{1}\right)\left(2 a+(n-i) \epsilon_{1}\right)}= \\
=\frac{(-)^{n}}{n !\left(\epsilon_{1} \epsilon_{2}\right)^{n}} \prod_{i=1}^{n} \frac{\epsilon^{2 n} \epsilon_{1}^{2 n}\left(\Delta_{0}+\Delta_{1}-\Delta_{2}+(i-1)\right)\left(\Delta_{0}+\Delta_{3}-\Delta_{4}+(i-1)\right)+O\left(\epsilon_{1}\right)}{\left(2 \alpha_{0}+(n-i) \epsilon_{1}\right)\left(2 \alpha_{0}-\epsilon+(n-i) \epsilon_{1}\right)} \longrightarrow \\
\longrightarrow \frac{1}{n !} \prod_{i=1}^{n} \frac{\left(\Delta_{0}+\Delta_{1}-\Delta_{2}+(i-1)\right)\left(\Delta_{0}+\Delta_{3}-\Delta_{4}+(i-1)\right)}{2 \Delta_{0}+(n-i)}
\end{array}
$$

The sum

$$
\sum_{n=0}^{\infty} x^{n} Z_{\left[1^{n}\right][0]} \longrightarrow \sum_{n=0}^{\infty} \frac{x^{n}}{n !} \prod_{k=0}^{n-1} \frac{\left(\Delta+\Delta_{1}-\Delta_{2}+k\right)\left(\Delta+\Delta_{3}-\Delta_{4}+k\right)}{2 \Delta+k}
$$

which exactly coincides with (3).

\subsection{Extra comments}

Some additional comments are in order. First of all, note that one can repeat the procedure of this paper in the case of $\epsilon_{2} \rightarrow 0$. Then, since the exchange $\epsilon_{1} \leftrightarrow \epsilon_{2}$ permutes rows and columns in the Young diagrams in the Nekrasov functions, one represents the same conformal block (39) as a sum of the anti-chiral partition functions $Z_{[n][0]}$.

Similarly, one can consider alternative possibility of keeping dimensions finite in the limit of either $\epsilon_{1} \rightarrow 0$ or $\epsilon_{2} \rightarrow 0$. Let, say, $\epsilon_{1} \rightarrow 0$. Then instead of (11) one can demand that $\epsilon-\alpha_{i} \sim \epsilon_{1}$, namely that

$$
\alpha_{i}=\epsilon-\epsilon_{1} \Delta_{i}+O\left(\epsilon_{1}^{2}\right)
$$

All above reasoning works in this case, only the two Young diagrams in $Z_{[Y],\left[Y^{\prime}\right]}$ interchanged, $Y \leftrightarrow Y^{\prime}$. If one imposes (40) in the limit of $\epsilon_{2} \rightarrow 0$ the Young diagrams will be permuted and transposed (the rows and columns exchanged).

In this paper, we considered the limit when the central charge of conformal theory goes to infinity, while all the dimensions remain finite. Instead, one could consider the limit when all the dimensions go to infinity along with the central charge. This is provided just by $\epsilon_{1} \rightarrow 0$ without adjustment of $\alpha_{i}$. To keep conformal blocks finite one should simultaneously take $x \rightarrow 0$. This limit is much more complicated since the Nekrasov functions corresponding to several Young diagrams at each level contribute in this case. Similarly, the analyzes of the conformal side is more involved in this case: it describes the quasiclassical limit of conformal theory. It is related to monodromy properties of an ordinary differential equation [16, 30. A particular case of conformal 
theory when all $\Delta_{i}$ remain finite but $\Delta_{0}$ (which is already quite involved) was considered in [30], the AGT relations in this case are studied in [14].

Note that the procedure presented here is immediately continued to higher orders in $\epsilon_{1}$ : one can construct a perturbation theory and check the AGT relation order by order in $\epsilon_{1}$, but in all orders in $x$. This is completely different from the standard checks of the AGT relation order by order in $x$ but in all orders in $\epsilon_{1}$, which were performed in [1, 2, 4, 8.

The limit $\epsilon_{1} \rightarrow 0$ corresponds to raising regularization of the moduli space integral in the instanton sector, while preserving it for antïnstantons. In result the integral over the instanton moduli space diverges. Within the "physical" prescription of refs. 35, 31], the divergency is compensated by subtracting a contribution from the boundary of the moduli space. This, however, breaks the symmetry of Nekrasov instanton calculus and, as we now understand, violates the AGT relations, the ones which would supposedly provide a solid group theory basis for a careful definition of appropriate $\tau$-functions. In this letter we suggested to make instanton contribution finite without subtractions, by taking instead a "double scaling" limit (11). Of course, in this case instantons continue to dominate over anti-instantons, so that actually only the instanton contribution survives in the form of the chiral Nekrasov functions. The answer, the hypergeometric series implies that the integral over the instanton moduli space satisfies a second-order differential equation w.r.t. $x$, what is unusual for naive $\mathrm{RG}$ equations, but quite normal for the exact RG group [36. It would be interesting to find a relation of this equation to the first-order RG equations in Seiberg-Witten theory [37, to understand its origin directly in terms of the instanton calculus, and, probably, even to extend this relation to the $\epsilon_{1} \neq 0$ region.

\section{Conclusion}

In this paper we proved the AGT relation for the Virasoro conformal blocks in the limit of large central charge c. The proof is not conceptual, but simply uses explicit formulas for both sides of the relation, which can be easily found in this limit. In fact, it turns out that the limit $c \rightarrow \infty$ imposes constraints on the conformal blocks and Nekrasov functions which appear similar to those imposed by the choice of the special conditions for external states, analyzed in [7, 8, the 4-point conformal block becomes hypergeometric and decomposes into a sum of the chiral Nekrasov functions for diagrams $\left(\left[1^{n}\right], \emptyset\right)$. Anyhow, this fact allows one to extend the existing proof of the AGT relations from special to generic external states. Still, it is restricted to the hypergeometric series, what is now achieved by imposing restriction on the central charge.

\section{Acknowledgements}

The work was partly supported by Russian Federal Nuclear Energy Agency and by RFBR grants 07-02-00878 (A.Mir.), and 07-02-00645 (A.Mor.). The work was also partly supported by joint grants 09-02-90493-Ukr, 09-02-93105-CNRSL, 09-01-92440-CE, 09-02-91005-ANF and by Russian President's Grant of Support for the Scientific Schools NSh-3035.2008.2.

\section{References}

[1] L.Alday, D.Gaiotto and Y.Tachikawa, arXiv:0906.3219

[2] N.Wyllard, arXiv:0907.2189

[3] N.Drukker, D.Morrison and T.Okuda, arXiv:0907.2593

[4] A.Marshakov, A.Mironov and A.Morozov, arXiv:0907.3946

[5] D.Gaiotto, arXiv:0908.0307

[6] Andrey Mironov, Sergey Mironov, Alexei Morozov and Andrey Morozov, arXiv:0908.2064

[7] A.Mironov and A.Morozov, Phys.Lett. B680 (2009) 188-194, arXiv:0908.2190

[8] A.Mironov and A.Morozov, arXiv:0908.2569

[9] S.Iguri and C.Nunez, arXiv:0908.3460

[10] D.Nanopoulos and D.Xie, arXiv:0908.4409 
[11] L.Alday, D.Gaiotto, S.Gukov, Y.Tachikawa and H.Verlinde, arXiv:0909.0945

[12] N.Drukker, J.Gomis, T.Okuda and J.Teschner, arXiv:0909.1105

[13] A.Marshakov, A.Mironov and A.Morozov, arXiv:0909.2052

[14] A.Marshakov, A.Mironov and A.Morozov, arXiv:0909:3338

[15] R.Dijkgraaf and C.Vafa, arXiv:0909.2453

[16] A.Belavin, A.Polyakov, A.Zamolodchikov, Nucl.Phys., B241 (1984) 333-380

[17] Al.Zamolodchikov and A.Zamolodchikov, Conformal field theory and critical phenomena in 2d systems, 2009, 168 p. (in Russian)

[18] P.Di Francesco, P.Mathieu and D.Sénéchal, Conformal Field Theory, Springer, 1997

[19] N.Nekrasov, Adv.Theor.Math.Phys. 7 (2004) 831-864

[20] R.Flume and R.Pogossian, Int.J.Mod.Phys. A18 (2003) 2541

[21] A.Losev, A.Marshakov and N.Nekrasov, in Ian Kogan memorial volume From fields to strings: circumnavigating theoretical physics, 581-621; hep-th/0302191

[22] N.Nekrasov and A.Okounkov, hep-th/0306238

[23] A.Marshakov and N.Nekrasov, JHEP 0701 (2007) 104, hep-th/0612019

[24] H.Nakajima and K.Yoshioka, math/0306198, math/0311058

[25] S.Shadchin, SIGMA 2 (2006) 008, hep-th/0601167

[26] D. Bellisai, F. Fucito, A. Tanzini and G. Travaglini, Phys. Lett. B 480 (2000) 365 hep-th/0002110

U.Bruzzo, F.Fucito, A.Tanzini, G.Travaglini, Nucl.Phys. B611 (2001) 205-226, hep-th/0008225

U.Bruzzo, F.Fucito, J.Morales and A.Tanzini, JHEP 0305 (2003) 054, hep-th/0211108

U.Bruzzo and F.Fucito, Nucl.Phys. B678 (2004) 638-655, math-ph/0310036

[27] F.Fucito, J.Morales and R.Pogossian, JHEP, 10 (2004) 037, hep-th/040890

[28] A. Mironov, A. Morozov and L. Vinet, Teor.Mat.Fiz. 100 (1994) 119-131 (Theor.Math.Phys. 100 (1995) 890-899), hep-th/9312213

A.Gerasimov, S.Khoroshkin, D.Lebedev, A.Mironov and A.Morozov, Int.J.Mod.Phys. A10 (1995) 25892614, hep-th/9405011;

S.Kharchev, A.Mironov and A.Morozov, q-alg/9501013;

A.Mironov, hep-th/9409190 Theor.Math.Phys. 114 (1998) 127, q-alg/9711006

[29] N.Seiberg and E.Witten, Nucl.Phys., B426 (1994) 19-52; Nucl.Phys., B431 (1994) 484-550

[30] Al.Zamolodchikov, Theor.Math.Phys. 73 (1987) 1088

[31] N.Dorey, T.Hollowood, V.Khoze and M.Mattis, Phys.Rept. 371 (2002) 231-459, arXiv:hep-th/0206063

[32] V.Fateev and I.Litvinov, JHEP 0711 (2007) 002, arXiv:0709.3806

[33] Al.Zamolodchikov, Comm.Math.Phys. 96 (1984) 419

[34] A.Belavin, private communication

[35] N.Dorey, V.Khoze and M.Mattis, Phys.Rev., D54 (1996) 7832-7848, hep-th/9607202 Nucl.Phys., B492 (1997) 607-622, hep-th/9611016

[36] J.Polchinski, Nucl.Phys., B231 (1984) 269

A.Mironov and A.Morozov, Phys.Lett. B490 (2000) 173-179

H.Sonoda, arXiv:0710.1662

[37] A.Gorsky, A.Marshakov, A.Mironov and A.Morozov, Nucl.Phys., B527 (1998) 690-716, hep-th/9802004

J.Edelstein, M.Marino and J.Mas, Nucl.Phys., B541 (1999) 671-697, hep-th/9805172

J.Edelstein, M.Gomez-Reino and M.Marino, Adv.Theor.Math.Phys., 4 (2000) 503-543, hep-th/0006113 